Conclusion The study suggests the association of hMR in sexual transmission of HIV. Presence of hMR in lower number of vaginal epithelial cells of Serodiscordant females prevented binding and HIV entry into these cells.

\section{P5.12 KNOWLEDGE AND AWARENESS AMONG CERVICAL CANCER PREVENTING VACCINE AMONG WOMEN IN SUBURBAN AREAS IN SRI LANKA}

Eashaan Maneendra Dassanayaka, EASI Edrisinghe, KAMJ Perera, WGWGC Bandara, SN Silva. International Institute of Health Sciences, Sri Lanka

\subsection{6/sextrans-2017-053264.628}

Introduction Cervical cancer is the 2nd most common cancer in the world whilst the incidence of cervical cancer continue to rise in Sri Lanka. It's important to assess the knowledge and the awareness on the cancer and the HPV vaccine. The Objective of this research was to assess the knowledge and awareness about the cervical cancers and HPV vaccine.

Methods A descriptive cross sectional study was done on 326 Sri Lankan urban and sub-urban females using convenient sampling who were in the age between 14-39 years using a self-administered questioner.

Results $(62.9 \%)$ of the participants, were from the age group 21-29. (50.8\%) had an advance level education, 30.4\% had an undergraduate level education. (55\%) haven't heard about a vaccine which could prevent cervical cancer, 51.2\% knows that cervical cancers are common in Sri Lanka. 193 (59.2\%) did not know that HPV is the most common cause for cervical cancers. When considering occupational level $(p<0.001)$ and and education level $(\mathrm{p}=0.001)$ it played a key role in determining the awareness of HPV vaccine on women. Respondents from the government sector with a good education had more awareness on the HPV vaccine. 28\% obtained information on HPV vaccine through health care professionals or the Internet. Only 4\% have been vaccinated and reason for not getting a vaccine is due to lack of knowledge. Only $11.3 \%$ knew about the correct does for the vaccine.

Conclusion It's evident that the knowledge and awareness is very low in terms of the vaccine.

\section{P5.13 WILLINGNESS TO USE PRE-EXPOSURE PROPHYLAXIS AMONG FEMALE SEX WORKERS IN THE MEXICO- UNITED STATES BORDER REGION}

${ }^{1}$ Heather Pines, ${ }^{1}$ Strathdee AS, ${ }^{2}$ Hendrix CW, Bristow CC, ${ }^{3}$ A Harvey-Vera, ${ }^{1}$ MagisRodríguez C, ${ }^{4}$ Martinez G, ${ }^{1}$ Semple SJ, ${ }^{1}$ Patterson TI. ' University of California, San Diego, USA; ${ }^{2}$ Johns Hopkins University, USA; ${ }^{3}$ Centro Nacional Para La Prevención y Control Del VIHISIDA (CENSIDA), Ministry of Health, Mexico; ${ }^{4}$ Federacion Mexicana de Asociaciones Privadas, Mexico

\subsection{6/sextrans-2017-053264.629}

Introduction We assessed willingness to use pre-exposure prophylaxis (PrEP) to prevent HIV among female sex workers (FSWs) in Tijuana and Ciudad Juarez, two northern Mexico cities bordering the United States (US).

Methods To date (7/2016-12/2016), 279 HIV-negative FSWs in a behavioural HIV prevention intervention trial have tested for sexually transmitted infections (STIs) and completed surveys to assess (1) preferences between and willingness to use 12 hypothetical PrEP products with varying attributes with respect to formulation (pill, gel, douche, or ring), frequency of use, cost, effectiveness, side effects, and access point and (2) perceived motivators and barriers to PrEP use. Fisher's exact tests were used to examine differences in willingness to use preferred PrEP products by STI diagnosis and sociodemographic, behavioural, and sex work characteristics.

Results 94\% (263/279) of FSWs were willing to use their preferred PrEP product. Willingness to use preferred PrEP products was lower among FSWs diagnosed with an STI $(83 \%$ vs. $97 \% ; \mathrm{p}=0.001)$, living in Tijuana (88\% vs. 99\%p<0.0001), primarily practicing sex work on the street $(89 \%$ vs. $97 \%$; $\mathrm{p}=0.01)$, reporting methamphetamine use (past month) $(86 \%$ vs. $97 \% ; \mathrm{p}=0.001)$, and reporting hazardous alcohol consumption (past year) $(91 \%$ vs. $97 \% ; \mathrm{p}=0.04)$. Of the 16 FSWs unwilling to use PrEP, "PrEP does not provide full protection against HIV" was the most common perceived barrier to PrEP use $(88 \%)$ followed by "I would have trouble using PrEP consistently because of my alcohol use" (63\%) and "I am worried I will lose clients if I use PrEP because they will assume I am HIV-positive" (63\%). All FSWs unwilling to use PrEP reported that additional protection against other STIs would motivate them to use PrEP.

Conclusion PrEP interest was high among FSWs along the Mexico-US border. Our findings suggest that the development of multi-purpose PrEP products and site-specific interventions that provide PrEP education and address substance use and clients' perceptions as barriers to PrEP use may support FSWs' future PrEP uptake.

\section{P5.14 AUTOIMMUNE DISEASES IN HIV-INFECTED PATIENTS}

1,2 Hejer Harrabi, 1,2Sameh Aissa, 1,2Rim Abdelmalek, ${ }^{2}$ Badreddine Kilani, 1,2 Lamia Ammari, ${ }^{2}$ Fakher Kanoun, ${ }^{1,2}$ Ahmed Ghoubontini, ${ }^{1,2}$ Hanene Tiouiri Benaissa. 'La Rabta University Hospital, Tunisia; ${ }^{2}$ Tunis- El Manar University, Tunisia

\subsection{6/sextrans-2017-053264.630}

Introduction Autoimmune and systemic diseases (ADs) were described in HIV infected patients and a classification by immune status was proposed.

Methods HIV-infected patients that presented an $\mathrm{AD}$ in the infectious diseases department of La Rabta University hospital in Tunis (Tunisia) were retrospectively included.

Results Four patients were included. The ADs were spondyloarthropathy, Behçet disease and psoriasis. Two patients presented Behçet disease. In two patients, the AD preceded HIV infection and in the two others, HIV infection was diagnosed at the same time as the $\mathrm{AD}$. In all cases, $\mathrm{ADs}$ occurred in patients with a CD4 T lymphocyte count of more than 200/ $\mathrm{mm}^{3}$. No co-infection with hepatitis $\mathrm{B}$ or $\mathrm{C}$ viruses was diagnosed.Three patients received anti-inflammatory drugs and one patient received immunosuppressant treatment with good tolerance.

Conclusion $\mathrm{AD}$ and autoantibodies are present in HIV infection. AD may develop during acute viral infection (Stage I), with normal to low CD4 counts (Stage II). However, past a threshold where the CD4 count is profoundly low, AD cannot develop (Stage III). Following HAART, immune restoration (normal CD4 count) with possible altered immune regulation may lead to the emergence of AD (Stage IV). More studies are necessary to identify the subgroups of HIV-infected patients that may be prone to develop AD. Co-infection with hepatitis $\mathrm{B}$ or $\mathrm{C}$ viruses should be screened. 